\title{
Case report: the etiology of anterior inferior cerebellar artery infarction: what does basi-parallel anatomic scanning magnetic resonance imaging tell us?
}

\author{
Zhi-yong Zhang $^{1 *+} \mathbb{D}$, Zhi Zhou ${ }^{2+}$, Hai-bo Zhang ${ }^{3}$ and Jin-song Jiao ${ }^{2}$
}

\begin{abstract}
Background: The precise etiology of anterior inferior cerebellar artery (AICA) infarction is difficult to identify because of the high anatomic variability of vertebrobasilar arteries and the limitations of conventional vascular examinations. Basi-parallel anatomic scanning magnetic resonance imaging (BPAS-MRI) can reveal the outer contour of the intracranial vertebrobasilar arteries, which may be helpful to distinguish the arteriosclerosis from congenital dysplasia and dissection.
\end{abstract}

Case presentation: In this study, we reported 3 cases of AICA infarction and discussed the diagnostic value of BPASMRI in the evaluation of vascular etiology.

Conclusions: The BPAS-MRI could be considered as an important supplementary in the diagnosis of vascular etiology of infarction in AICA territory.

Keywords: Etiology, Anterior inferior cerebellar artery, Stroke, Vertebrobasilar artery, Basi-parallel anatomic scanning magnetic resonance imaging

\section{Background}

The anterior inferior cerebellar artery (AICA) infarction may cause vertigo, hearing loss, facial weakness, Horner's syndrome, ataxia or other neurological symptoms. As the treatments might vary due to different etiologies, the accurate identification of etiology is of great significance to the management. Conventional vascular imaging methods, including the time-of-flight magnetic resonance angiography (TOF-MRA) and computed tomographic angiography (CTA), have limitations in the diagnosis of AICA's own lesions. First, AICA is not

\footnotetext{
*Correspondence: zhiyong_zhang888@126.com

†Zhi-yong Zhang and Zhi Zhou contributed equally to this work.

1 Department of Neurology, Beijing Geriatric Hospital, 118 Wenquan

Road, Haidian District, Beijing 100095, China

Full list of author information is available at the end of the article
}

typically visualized on CTA due to the limited detection of the smaller branches [1]. Second, as these methods can only show the narrowing or absence of an artery with the illustration of inner vascular wall, it's difficult to distinguish the stenosis or occlusion from hypoplasia or aplasia because of the high anatomic variability in vertebrobasilar artery system [2]. Therefore, due to the uncertainty of AICA displayed by routine vascular examination, the etiology of AICA infarction is more confusing. Basi-parallel anatomic scanning magnetic resonance imaging (BPASMRI) can reveal the outer contour of the vertebrobasilar arteries and has good value for differential diagnosis in atherosclerotic stenosis, hypoplasia and dissection, which may be of help for the diagnosis of AICA lesions [3, 4]. We herein present 3 cases with AICA infarction and 
discuss the value of BPAS-MRI in the diagnosis of vascular etiology of AICA infarction.

\section{Case presentation}

\section{Case 1}

A 69-year-old woman with history of diabetes mellitus and hypertension presented with sudden vertigo, left hearing loss, and left ataxia. MRI showed hyperintensity in left brachium pontis and left superior lateral cerebellum on diffusion-weighted image (DWI) (Fig. 1a), indicating an acute infarction in left AICA territory. CTA showed the server stenosis and occlusion of left veterbral artery and absence of bilateral AICA (Fig. 1b). Digital subtraction angiography (DSA) showed occlusion from left proximal AICA (Fig. 1c). Combined with the history, the etiology was presumed as atherosclerotic occlusion of left AICA.

\section{Case 2}

A 64-year-old male patient with hypertension presented with vertigo, left peripheral facial palsy and left ataxia. DWI revealed left AICA territorial infarction (left inferolateral pons, brachium pontis, superior cerebellum) (Fig. 2a). On MRA, the left AICA was absent, while the right AICA was visualized clearly (Fig. 2b). BPAS-MRI showed normal bilateral AICA (Fig. 2c). Therefore, the radiological diagnosis of the left AICA occlusion was made, and left AICA was the victim vessel of this infarction.

\section{Case 3}

A 60-year-old male patient presented with vertigo and right ataxia. The DWI showed acute infarction involving right inferolateral pons, brachium pontis and superior cerebellum (Fig. 3a). The right AICA was absent on MRA (Fig. 3b) and BPAS-MRI (Fig. 3c). The comprehensive diagnosis was considered as right AICA congenital aplasia, and other causes of the infarction needed to be explored. Finally, a proximal atrial fibrillation was found through long-term monitoring, and the cardiac embolism was presumed as the etiology of infarction.
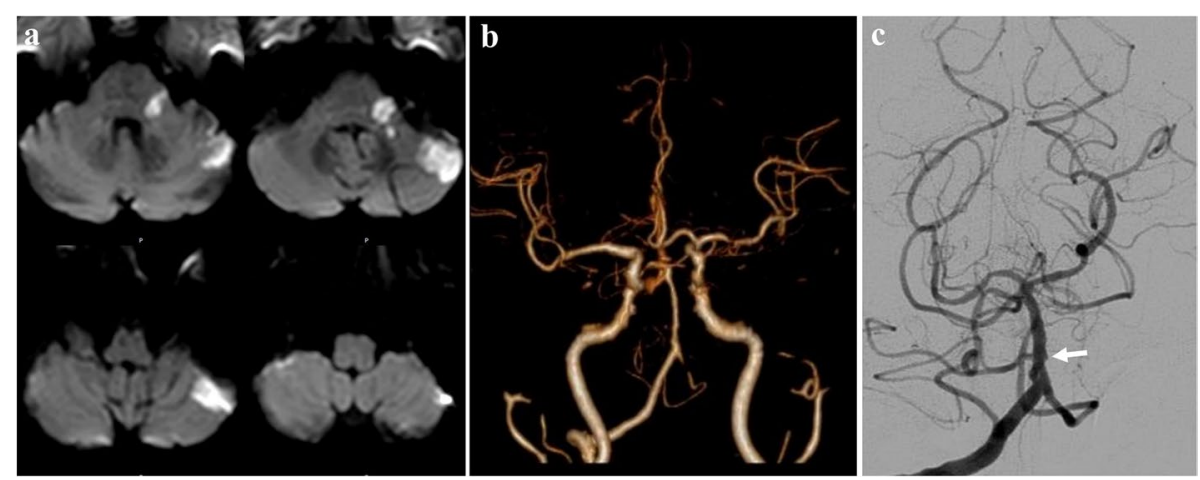

Fig. 1 Diffusion-weighted image revealed acute infarction on left brachium pontis and left superior lateral cerebellum (a). Computed tomographic angiography showed the server stenosis and occlusion of left veterbral artery and the absence of bilateral anterior inferior cerebellar artery (AICA) (b). Digital subtraction angiography showed occlusion from left proximal AICA (c)

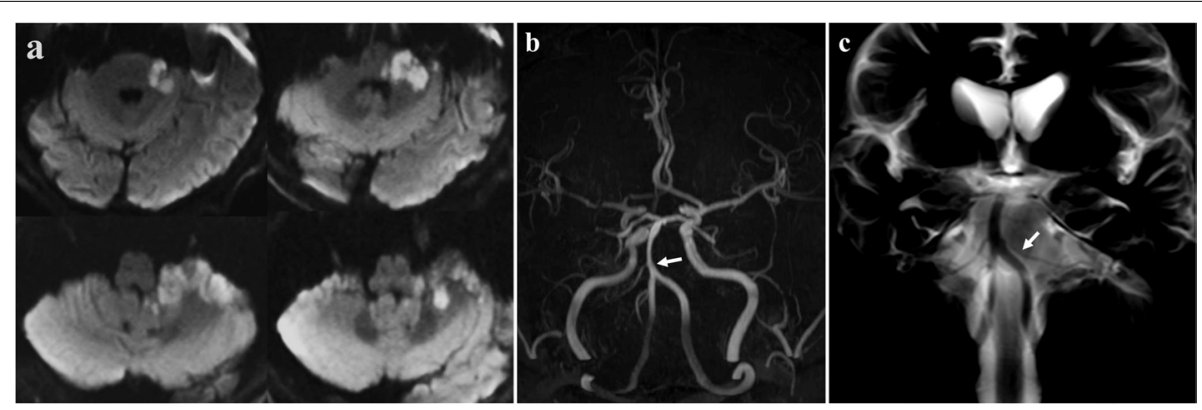

Fig. 2 Diffusion-weighted image revealed left anterior inferior cerebellar artery (AICA) territorial infarction (a).On magnetic resonance angiography, the left AICA was absent, while the right AICA was visualized clearly (b). Basi-parallel anatomic scanning magnetic resonance imaging (BPAS MRI) showed normal bilateral AICA (c) 

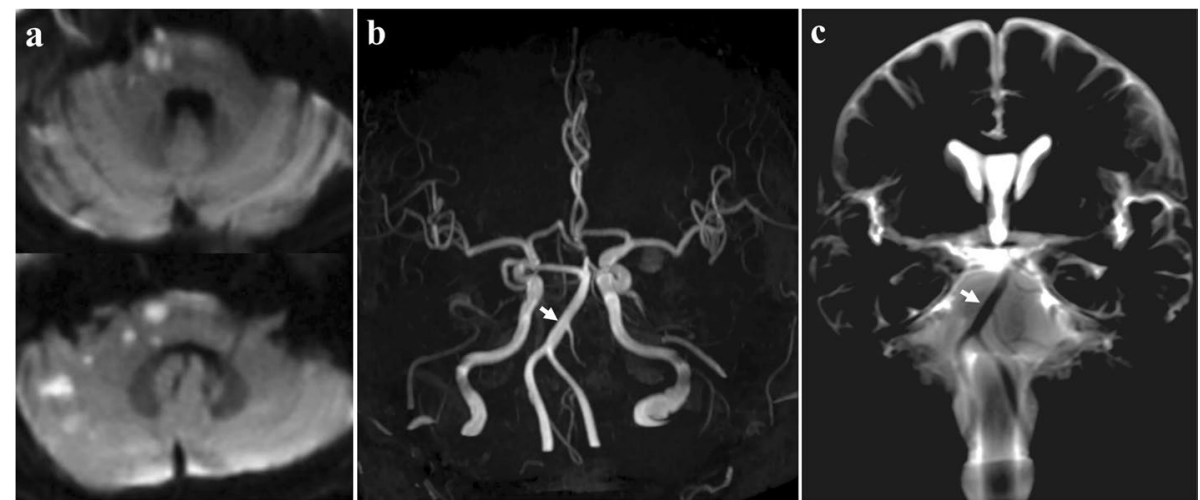

Fig. 3 The diffusion-weighted image showed hyperintensity lesions in right inferolateral pons, brachium pontis and superior cerebellum (a). The right anterior inferior cerebellar artery (AICA) was absent on magnetic resonance angiography (b) and basi-parallel anatomic scanning magnetic resonance imaging (BPAS-MRI) (c)

\section{Discussion}

The etiology of AICA infarction is difficult to diagnose due to the high anatomic variability in vertebrobasilar system, even both of the patients in Case 1 and Case 2 had atherosclerotic risk factors in our study. It's reported that only $35 \%$ of patients had normal vestebrobasilar arteries, and approximately $29 \%$ of the posterior circulation anatomic variability is related to AICA [5]. In Case 1, if the occlusion was from the origin of AICA instead of the proximal part, even the DSA, which has widely been considered as the gold standard, still could not differentiate the occlusion from congenital dysplasia.

Compared with conventional methods, such as TOFMRA, CTA or DSA, BPAS-MRI could provide an accurately visualization of the outer contour of vascular wall regardless the influence of blood flow or thrombus, even when the artery is occluded. In Case 2, the absent of left AICA on TOF-MRA and the presence of AICA on BPAS-MRI imaging led to a straightforward diagnosis of AICA occlusion due to atherosclerosis or thrombus. This suggested the supplementary role of BPAS-MRI to TOF-MRA in the diagnosis of AICA occlusion. In Case 3, though the topography of infarction was consistent with AICA territory, the absence of AICA on MRA and BPAS-MRI indicated the congenital aplasia of AICA. If we simply attributed the cause to AICA occlusion, we might miss the real etiology and take inappropriate treatment.

In the future, large-scale case studies with multiple imaging modalities are needed to validate the supplementary role of BPAS-MRI in the diagnosis of etiology of AICA.

\section{Conclusions}

The BPAS-MRI could be considered as an important supplementary in the diagnosis of vascular etiology of infarction in AICA territory.

\section{Abbreviations \\ AICA: Anterior inferior cerebellar artery; TOF-MRA: Time-of-flight magnetic resonance angiography; CTA: Computed tomographic angiography; BPAS- MRI: Basi-parallel anatomic scanning magnetic resonance imaging; DWI: Diffusion-weighted image; DSA: Digital subtraction angiography.}

\section{Acknowledgements}

The authors thank the MRI technologists and operators for their assistance, and thank all the participants for their dedication, time, and effort to this study.

\section{Authors' contributions}

Conceptualization: ZZ1 and JJ; Methodology: HZ; Writing —original draft preparation: ZZ2; Writing — review and editing: ZZ1 and ZZ2; Revision: ZZ1 Supervision: ZZ1. ZZ1 would correspond to the author furthest up on the author list. All authors read and approved the final manuscript.

\section{Funding}

Not applicable.

\section{Availability of data and materials}

The data used during the current study are available from the corresponding author on reasonable request.

\section{Declarations}

\section{Ethics approval and consent to participate}

The study was approved by the Research Ethics Committees of China-Japan Friendship Hospital. All procedures were performed in accordance with methods approved by the Ethics Committee.

\section{Consent for publication}

Written informed consent was obtained from each of the patient for publication of this Case report with their personal or clinical details and any accompanying images. A copy of the written consent is available for review by the Editor of this journal. 


\section{Competing interests}

The authors declare no competing interests.

\section{Author details}

${ }^{1}$ Department of Neurology, Beijing Geriatric Hospital, 118 Wenquan Road, Haidian District, Beijing 100095, China. ²Department of Neurology, ChinaJapan Friendship Hospital, Beijing 100029, China. ${ }^{3}$ Department of Radiology, China-Japan Friendship Hospital, Beijing 100029, China.

Received: 18 August 2020 Accepted: 6 July 2021

Published online: 30 July 2021

\section{References}

1. Amarenco P, Rosengart A, DeWitt LD, Pessin MS, Caplan LR. Anterior inferior cerebellar artery territory infarcts. Mechanisms and clinical features. Arch Neurol. 1993;50(2):154-61.

2. Akgun V, Battal B, Bozkurt Y, Oz O, Hamcan S, Sari S, Akgun H. Normal anatomical features and variations of the vertebrobasilar circulation and its branches: an analysis with 64-detector row CT and 3T MR angiographies. ScientificWorldJournal. 2013;2013:620162.

3. Nagahata M, Abe Y, Ono S, Hosoya T, Uno S. Surface appearance of the vertebrobasilar artery revealed on basiparallel anatomic scanning (BPAS)MR imaging: its role for brain MR examination. AJNR Am J Neuroradiol. 2005;26(10):2508-13

4. Iwata R, Yamashita K, Nishikawa T, Kajiwara M, Ueba T. Diagnosis of anterior inferior cerebellar artery occlusion on magnetic resonance angiography with reference to basiparallel anatomic scanning-magnetic resonance imaging. Neurol Med Chir. 2010;50(11):987-9.

5. Chen MM, Chen SR, Diaz-Marchan P, Schomer D, Kumar VA. Anterior inferior cerebellar artery strokes based on variant vascular anatomy of the posterior circulation: clinical deficits and imaging territories. J Stroke Cerebrovasc Dis. 2018:27(4):e59-64.

\section{Publisher's Note}

Springer Nature remains neutral with regard to jurisdictional claims in published maps and institutional affiliations.
Ready to submit your research? Choose BMC and benefit from:

- fast, convenient online submission

- thorough peer review by experienced researchers in your field

- rapid publication on acceptance

- support for research data, including large and complex data types

- gold Open Access which fosters wider collaboration and increased citations

- maximum visibility for your research: over $100 \mathrm{M}$ website views per year

At BMC, research is always in progress.

Learn more biomedcentral.com/submissions 